\title{
Ozone and Other 1,3-Dipoles: Toward a Quantitative Measure of Diradical Character
}

\author{
Benoît Braïda, * ${ }^{\dagger}$ Sérgio E. Galembeck, ${ }^{\ddagger}$ and Philippe C. Hiberty ${ }^{*}$, \\ †UPMC Université Paris 06, CNRS UMR 7616, Laboratoire de Chimie Théorique, Case Courrier 137, 4 Place Jussieu, 75252 Paris, \\ France \\ ${ }^{\ddagger}$ Departamento de Química, Faculdade de Filosofia, Ciências e Letras de Ribeirão Preto, Universidade de São Paulo, 14040-901, \\ Ribeirão Preto - SP Brazil \\ ${ }^{\S}$ Laboratoire de Chimie Physique, Groupe de Chimie Théorique, CNRS UMR 8000, Université de Paris-Sud, 91405 Orsay Cédex, \\ France
}

ABSTRACT: Ozone and its sulfur-substituted isomers are studied by means of the Breathing Orbital Valence Bond ab initio method, with the objective of estimating their controversial diradical characters. The calculated weights of the various VB structures and their individual diabatic energies are found to be consistent with each other. All 1,3dipoles can be described in terms of three major VB structures, one diradical and two zwitterionic ones, out of the six structures, forming a complete basis. Ozone has a rather large diradical character, estimated to $44 \%-49 \%$. SOO and SOS are even more diradicalar, whereas SSO

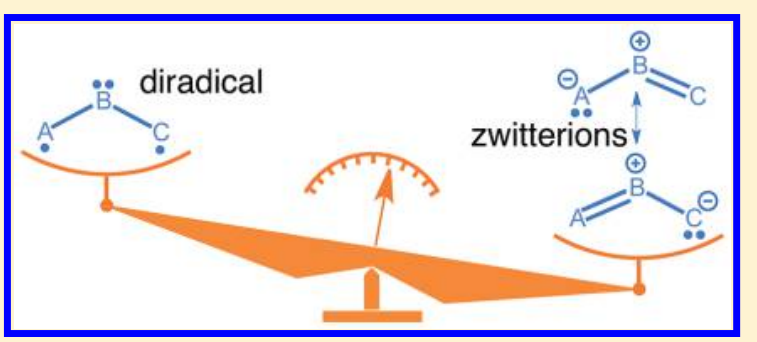
and especially OSO are better described as closed-shell zwitterions. Moreover, the description of 1,3-dipoles, in terms of the three major structures, yields VB weights in full agreement with simple chemical wisdom, i.e., a diradical weight of $33 \%$ when the three structures are quasi-degenerate, and a smaller (larger) value when the diradical structure is higher (lower) in energy than the zwitterionic ones. Therefore, the VB-calculated weight of the diradical structure of a molecule qualifies itself as a quantitative measure of diradical character, and not only as an indicator of tendencies. Other definitions of the diradical character, based on molecular orbital/configuration interaction methods, are discussed.

\section{INTRODUCTION}

Ozone and other isoelectronic 1,3-dipoles are powerful synthetic reagents, because of their ability to react with alkenes and alkynes to undergo $3+2$ concerted cycloadditions, leading to five-membered rings. ${ }^{1,2}$ They all share similar electronic structures, with four electrons occupying three $\pi$-type valence orbitals, and, therefore, they can be described in valence bond terms with the same set of Lewis structures, but with different weights. Among 1,3-dipoles, ozone has been, by far, one studied the most often, because of its immense importance in chemical industry and its presence in the troposphere and stratosphere.

Generally, a crucial property of 1,3-dipoles is their diradical characters, which, as will be discussed below, have significant repercussions on their reactivities and molecular properties. Therefore, it is very important to be able to estimate this aspect of their electronic structure as accurately as possible.

On the theoretical side, the research group of Hay, Dunning, and Goddard ${ }^{3-5}$ were first to perform generalized valence bond (GVB) calculations, showing the importance of the diradical structure $\mathbf{D}$ in the description of ozone in Lewis structure terms (see Scheme 1), despite the fact that this molecule was always (and still is) represented as a resonance between two zwitterionic structures $\mathrm{Z}_{1}$ and $\mathrm{Z}_{2}$ in most chemistry textbooks. ${ }^{6}$ Indeed, the shapes of the GVB orbitals pointed to a ground state closely resembling a diradical having two unpaired $\pi$ electrons on the terminal oxygens, weakly coupled into a singlet
Scheme 1. Diradical (D) and Main Zwitterionic $\left(\mathrm{Z}_{1}, \mathrm{Z}_{2}\right)$ Lewis Structures of Ozone

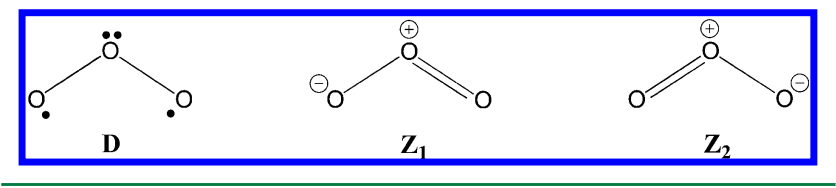

state. ${ }^{5}$ As another, more numerical argument, they noted that a closed-shell structure of ozone is not supported by ab initio calculations, since the single-determinant Hartree-Fock incorrectly places the ${ }^{3} \mathrm{~B}_{2}$ state some $3 \mathrm{eV}$ below the actual ${ }^{1} \mathrm{~A}_{1}$ ground state. ${ }^{4}$ Similarly, many theoreticians interpreted the well-established multireference character of the ground state of ozone and/or the small singlet-triplet energy gap as a proof of significant diradical character, ${ }^{3-5,7-12}$ a point of view that was however rejected by Kalemos and Mavridis. ${ }^{13}$ On the experimental side, it was the existence of several low-lying excited states of ozone that was considered as a proof of diradical character. ${ }^{14,15}$

Turning to other 1,3-dipoles, Dunning et al. compared OSO and $\mathrm{SOO}$ on the basis of GVB calculations and concluded that (i) SOO has substantial diradical character, similar to ozone, 
while OSO does not, and (ii) these contrasted natures of the two isomers explain their dramatic differences in structures and stabilities. ${ }^{10}$ The same authors also compared ozone to OSO and showed that the negligible diradical character of the latter, at variance with ozone, accounts for the differences of these two species, with regard to physical properties and reactivity toward the addition of hydrogen to their terminal atoms. ${ }^{11,12}$ Identical conclusions were reached by Cooper et al., using the spincoupled method, ${ }^{16}$ which is also known as full-generalized GVB. Thus, ozone was described as a combination of diradical and closed-shell structures, neither being dominant, whereas the closed-shell description was overwhelmingly dominant for $\mathrm{SO}_{2}{ }^{17}$

Complementary to these qualitative descriptions, many theoretical studies have attempted to quantify the diradical character of ozone and other 1,3-dipoles, by interpreting wave functions based on molecular orbitals (MOs) and calculated after configuration interaction (CI). These interpretations are based on the relative proportions of the Hartree-Fock configuration, $\varphi_{1}$, and the lowest diexcited one, $\varphi_{2}$, in the ground state wave function $\Psi\left({ }^{1} \mathrm{~A}_{1}\right)$ (see eqs $\left.1-3\right)$ :

$$
\begin{aligned}
& \varphi_{1}=\left(\ldots \pi_{0}{ }^{2} \pi_{1}{ }^{2}\right) \\
& \varphi_{2}=\left(\ldots \pi_{0}{ }^{2} \pi_{2}{ }^{2}\right) \\
& \Psi\left({ }^{1} A_{1}\right)=C_{1} \varphi_{1}+C_{2} \varphi_{2}+\ldots
\end{aligned}
$$

where $\pi_{0}, \pi_{1}$, and $\pi_{2}$ are the lowest-lying molecular orbitals (MOs) of $\pi$-type.

Assuming that a 50:50 mixture of $\varphi_{1}$ and $\varphi_{2}$ corresponds to a pure diradical, Laidig and Schaefer ${ }^{7}$ coined the $B$ index as a measure of the diradical character for electronic systems, such as ozone:

$$
B=2 C_{2}^{2}
$$

However, the so-estimated diradical character of ozone proved to be quite dependent on the size of the CI space, with $B$ values of $46 \%, 5 \%$, and $23 \%$ for MCSCF spaces involving 2, 6825, and 13413 configurations, respectively, ${ }^{7}$ and $26 \%$ in a CASSCF$(18,12)$ calculation by Tuononen et al. ${ }^{18}$

To remedy this shortcoming, Xantheas et al. ${ }^{19}$ proposed a normalized formula (yielding values always between 0 and 1) for parameter $B_{\mathrm{CI}}$ in eq 5 :

$$
B_{\mathrm{CI}}=\left[\frac{2 C_{2}{ }^{2}}{\left(C_{1}{ }^{2}+{C_{2}}^{2}\right)}\right]^{1 / 2}
$$

These authors applied this formula to $\operatorname{CASSCF}(18,12)$ calculations of four 1,3-dipoles and got a diradical character of $43.2 \%$ for ozone. The same authors also proposed another index, $B_{\text {orb}}$, based on the $\pi$ atomic orbital occupancies of the studied molecules. Thus, assuming that $\mathbf{D}, \mathbf{Z}_{1}$, and $\mathrm{Z}_{2}$ are the most important structures for ozone and isoelectronic systems, since the $\pi$-occupation of the central atom is 2.0 for the diradical structure $\mathbf{D}$ and 1.0 for $\mathbf{Z}_{1}$ and $\mathbf{Z}_{2}$, an intuitively attractive definition of the diradical character follows:

$$
B_{\text {orb }}=\pi \text {-occupation at the center atom }-1
$$

Remarkably, the $B_{\mathrm{CI}}$ and $B_{\text {orb }}$ indices were determined to be consistent for $\mathrm{O}_{3}$, SOS, and $\mathrm{S}_{3}$, less so for OSO. Later, Xantheas et al. ${ }^{20}$ abandoned the $B_{\mathrm{CI}}$ index and preferred to use its squared value ( $\beta$ in eq 7 below), which they deemed to be better justified theoretically:

$$
\beta=\frac{2 C_{2}{ }^{2}}{C_{1}{ }^{2}+C_{2}{ }^{2}}
$$

As a consequence, this new definition yielded diradical characters systematically smaller than $B_{\mathrm{CI}}$ for the studied dipoles, with a value of only $18.0 \%$ for ozone.

All these analyses of $\mathrm{MO}-\mathrm{CI}$ wave functions, using the $B$, $B_{\mathrm{CI}}$, and $\beta$ indices, rely on the assumptions that the HartreeFock configuration has no diradical character at all, whereas the 50:50 combination of Hartree-Fock and lowest diexcited configurations features a $100 \%$ diradical. While the latest assumption is perfectly true in some particular cases, like twisted ethylene $^{21}$ or trimethylenemethane, ${ }^{22}$ there is no indication that it carries over to 1,3-dipoles. Quite the contrary, some simple hand exercises consisting of rigorously expanding $\mathrm{MO}-\mathrm{CI}$ wave functions into valence bond (VB) ones, shows that (1) $\varphi_{1}$ contains some diradical character, even if minor, and (2) $\left(\varphi_{1}-\varphi_{2}\right)$ is not $100 \%$ radical but contains some minor ionic components in the ozone case. ${ }^{23}$ Now, instead of analyzing $\mathrm{MO}-\mathrm{CI}$ wave functions, why not use ab initio $\mathrm{VB}$ methods, which are able to directly quantify the coefficients and weights of each VB structure? The advantage of such methods is that, if the atomic orbitals are clearly defined as singlecentered on a given atom, there is no ambiguity as to the correspondence between the VB functions ("VB structures") and the Lewis structures such as $\mathbf{D}, \mathbf{Z}_{1}, \mathbf{Z}_{2}$, or others, for ozone.

Such calculations have been performed in the past for 1,3dipoles, either by transforming $\mathrm{MO}-\mathrm{CI}$ wave functions to $\mathrm{VB}$ ones, ${ }^{24}$ or by direct ab initio VB calculations, ${ }^{25}$ leading to consistent diradical weights for ozone $(47.6 \%$ and $49.5 \%$, respectively). Subsequent VB calculations were performed for three families of 1,3-dipoles: diazonium betaines, nitrilium betaines, and azomethine betaines. Within each family, the calculated diradical character was found to closely correlate with the activation barrier to cycloaddition, regardless of whether the addition was on ethylene or acetylene. ${ }^{26}$ Besides, other correlations, this time with molecular properties of ozone and its sulfur-substituted analogues, ${ }^{20}$ were also presented by Xantheas et al. by using the $\beta$ index, which, as we will show, yields values that vary almost linearly with VB values.

Now, even if the various indices are found at least to display some meaningful tendencies, is it possible to define a quantitative, or "absolute", diradical character of a molecule in general?

\section{CAN ONE DEFINE A QUANTITATIVE MEASURE OF DIRADICAL CHARACTER?}

As a method that directly expresses a wave function in terms of Lewis structures, VB theory appears to be ideally suited to estimate the weights of a diradical structure relative to the other VB structures in any molecule. Now, there remains to check if the so-calculated weights are only indicative numbers that can show tendencies in some series, or can be taken as accurate measures that (1) provide balanced weights of the various $\mathrm{VB}$ structures, and (2) are consistent with well-defined quantities, such as energy gaps.

In classical VB theory, a many-electron wave function is expressed as a linear combination of all of the VB structures that can be generated by distributing the electrons in the various atomic orbitals, as expressed in eq 8 : 


$$
\Psi_{\mathrm{VB}}=\sum_{K} C_{K} \Phi_{K}
$$

where each $\Phi_{K}$ corresponds to a classical VB structure defined with atomic orbitals (AOs) that are each strictly centered on a single atom (or fragment in the general case), at variance with semilocalized orbitals that are used in the GVB and SC families of VB methods. Of course, such strictly localized AOs are nonorthogonal. The coefficients $C_{K}$ are subsequently determined by solving the usual secular equation $H C=E M C$, where the Hamiltonian and overlap matrix elements are defined as

$$
H_{K L}=\left\langle\Phi_{K}|\hat{H}| \Phi_{L}\right\rangle
$$

and

$$
M_{K L}=\left\langle\Phi_{K} \mid \Phi_{L}\right\rangle
$$

In the VBSCF method of van Lenthe and Balint-Kurti, ${ }^{27}$ both $\mathrm{VB}$ orbitals and structure coefficients are simultaneously optimized to minimize the total energy, in strong analogy with the CASSCF method in the MO framework. Since the $\mathrm{MO}$ configurations and VB structures span the same space of configurations, the secular equations in the $\mathrm{MO}$ or $\mathrm{VB}$ framework, at this level of calculation, must lead to almost equivalent wave functions, albeit expressed in different forms. Thus, in the same way as CASSCF yields energies for the various configurations, $a b$ initio $\mathrm{VB}$ provides reliable energy gaps between VB structures, so that such quantities can safely be used as guides to control the validity of other indices, such as structural weights.

The most commonly used formula for calculating weights of VB structures from eq 8 is the Chirgwin-Coulson formula: ${ }^{28}$ :

$$
W_{K}=C_{K}^{2}+\sum_{L \neq K} C_{K} C_{L} M_{K L}
$$

Here, it is clear that the first term, $C_{K}^{2}$, entirely belongs to VB structure $\Phi_{K}$, whereas the second term is shared between $\Phi_{K}$ and $\Phi_{L}$, which makes the definition questionable when the overlap between $\Phi_{K}$ and $\Phi_{L}$ is large. To remedy this overlapdependence problem, Gallup and Norbeck proposed an alternative definition, ${ }^{29}$ called "inverse weights":

$$
W_{K}^{\mathrm{INV}}=\frac{N C_{K}{ }^{2}}{M_{K K}{ }^{-1}}
$$

where $N$ is a normalization factor. Both definitions (eqs 11 and 12) sum to unity (or $100 \%$, if one prefers). In practice, the weights computed using the two definitions do not usually depart significantly from each other when the overlap between VB structures is not too large, which is a condition that is met in the present case, since the active AOs, being of $\pi$-type, overlap only weakly.

Now, since weights of VB structures are not observable properties, it is difficult to assess their reliability in the general case. However, it turns out that the particular case of 1,3dipoles, particularly the symmetrical ones (e.g., $\mathrm{O}_{3}$, OSO, SOS, $\mathrm{S}_{3}, \ldots$ ), offers a way to check the balance between the weights of the various VB structures, and even to check whether these weights can be considered as absolute values or only relative ones. The demonstration is based on the fact that $\mathrm{ab}$ initio VB theory is able to provide not only the weights $W_{K}$ of the VB structures, but also their diabatic energies $E_{K}$. Furthermore, we can use the widely accepted simplifying assumption (that will be computationally checked later in this work) that 1,3-dipoles are well-described as a combination of three main VB structures, $\mathbf{D}, \mathbf{Z}_{1}$, and $\mathbf{Z}_{2}$ (see Schemes 1 and 2), whereas the three remaining ones contribute much less to the ground state.

\section{Scheme 2. The Six VB Structures of General 1,3-Dipoles}

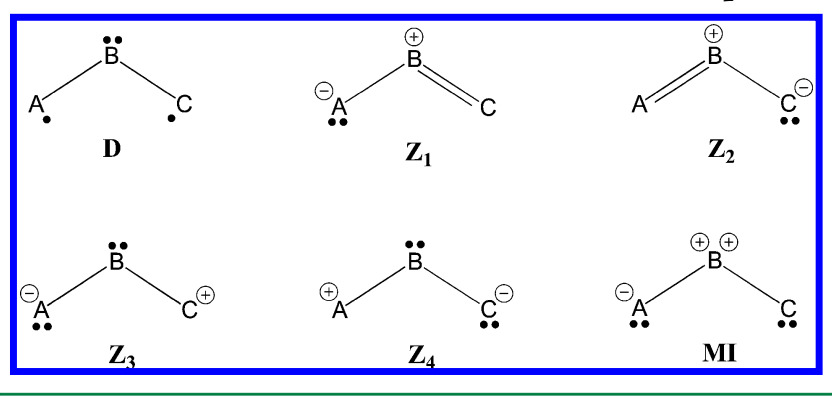

Focusing now on the three main VB structures, $\mathbf{Z}_{\mathbf{1}}$ and $\mathbf{Z}_{\mathbf{2}}$ are equivalent in a symmetrical 1,3-dipole and must therefore be degenerate and have identical weights $\left(E_{\mathrm{Z}_{1}}=E_{\mathrm{Z}_{2}}, W_{\mathrm{Z}_{1}}=W_{\mathrm{Z}_{2}}\right)$. Remembering that the weights sum to $100 \%$, they must obey the simple relation given below:

$$
W_{\mathrm{D}}+2 W_{\mathrm{Z} 1}=100 \%
$$

Now, since the VB structure that is lowest-lying in energy necessarily has the largest weight of the three, it follows that

$$
\begin{array}{ll}
W_{\mathrm{D}}>33.3 \% & \text { if } E_{\mathrm{D}}<E_{\mathrm{Z}_{1}} \\
W_{\mathrm{D}}<33.3 \% & \text { if } E_{\mathrm{Z}_{1}}<E_{\mathrm{D}}
\end{array}
$$

Finally, the weight of the diradical structure is expected to be close to $33.3 \%$ if the three VB structures have approximately the same energy:

$$
W_{\mathrm{D}} \approx 33.3 \% \quad \text { if } E_{\mathrm{D}} \approx E_{\mathrm{Z}_{1}} \approx E_{\mathrm{Z}_{2}}
$$

In view of the variety of values and opinions that have been published so far on the diradical characters of ozone and other 1,3 dipoles, and of the uncertainty, with regard to the absolute values of the diradical characters coming out of the corresponding definitions, we performed high-level $a b$ initio classical VB calculations on a series of 1,3-dipoles of very contrasted reactivities: $\mathrm{O}_{3}$, OSO, SOO, SSO, SOS, and $\mathrm{S}_{3}$. We will show that the VB-calculated weights of diradical structures are consistent with the relative energies of the various VB structures and satisfy eqs $13-15$, and therefore that these diradical weights can be taken as reference values for the diradical characters of 1,3-dipoles and other types of molecules in general.

\section{COMPUTATIONAL METHODS}

There are several computational approaches for VB theory at the $a b$ initio level. ${ }^{23}$ In the VB self-consistent-field (VBSCF) procedure, ${ }^{27}$ both the $\mathrm{VB}$ orbitals and structural coefficients are optimized simultaneously to minimize the total energy. The breathing-orbital valence bond method (BOVB) ${ }^{30}$ improves the VBSCF accuracy without increasing the number of VB structures $\Phi_{K}$. This is achieved by allowing each VB structure to have its own specific set of orbitals during the optimization process, such that the orbitals can be different from one VB structure to the other. In this manner, the orbitals can fluctuate in size and shape, to fit the instantaneous charges of the atoms on which these orbitals are located. 
Table 1. Relative Diabatic Energies of the Individual VB Structures, and Absolute Energies of the Multistructure Ground States

\begin{tabular}{|c|c|c|c|c|c|c|}
\hline & $\mathrm{O}-\mathrm{S}-\mathrm{O}$ & $\mathrm{O}-\mathrm{S}-\mathrm{S}$ & $S-S-S$ & $\mathrm{O}-\mathrm{O}-\mathrm{O}$ & $\mathrm{S}-\mathrm{O}-\mathrm{O}$ & $\mathrm{S}-\mathrm{O}-\mathrm{S}$ \\
\hline \multicolumn{7}{|c|}{ Diabatic Energy $\left(\mathrm{kcal} \mathrm{mol}^{-1}\right)$} \\
\hline $\mathrm{D} \cdot \mathrm{A}-\mathrm{B}-\mathrm{C}^{\bullet}$ & 0 & 0 & 0 & 0 & 0 & 0 \\
\hline $\mathrm{Z}_{1}{ }^{-} \mathrm{A}-\mathrm{B}^{+}=\mathrm{C}$ & -48.7 & -11.7 & +14.6 & +44.5 & +55.3 & +56.5 \\
\hline $\mathrm{Z}_{2} \mathrm{~A}=\mathrm{B}^{+}-\mathrm{C}^{-}$ & -48.7 & -3.2 & +14.6 & +44.5 & +48.5 & +56.5 \\
\hline $\mathrm{MI}^{-} \mathrm{A}-{ }^{+} \mathrm{B}^{+}-\mathrm{C}^{-}$ & +106.3 & +125.8 & +135.5 & +241.9 & & \\
\hline \multicolumn{7}{|l|}{$\mathrm{Z}_{3}{ }^{-} \mathrm{A}-\mathrm{B}-\mathrm{C}^{+}$} \\
\hline $\mathrm{Z}_{4}{ }^{+} \mathrm{A}-\mathrm{B}-\mathrm{C}^{-}$ & & +209.3 & & & & \\
\hline \multicolumn{7}{|c|}{ Ground-State Absolute Energy (Hartrees) } \\
\hline full BOVB & -547.35343 & -869.99930 & -1192.65171 & -224.48266 & -547.17906 & -869.90098 \\
\hline
\end{tabular}

Table 2. Coulson-Chirgwin Weights of the Individual VB Structures (from eq 11) and Inverse Weights (from eq 12, Given in Parentheses)

\begin{tabular}{lccccc} 
& $\mathrm{O}-\mathrm{S}-\mathrm{O}$ & $\mathrm{O}-\mathrm{S}-\mathrm{S}$ & $\mathrm{S}-\mathrm{S}-\mathrm{S}$ & $\mathrm{O}-\mathrm{O}-\mathrm{O}$ & $\mathrm{S}-\mathrm{O}-\mathrm{O}$ \\
$\mathrm{D}{ }^{\bullet} \mathrm{A}-\mathrm{B}-\mathrm{C}^{\bullet}$ & $0.159(0.150)$ & $0.227(0.257)$ & $0.346(0.377)$ & $0.444(0.486)$ & $0.561(0.578)$ \\
$\mathrm{Z}_{1}{ }^{-} \mathrm{A}-\mathrm{B}^{+}=\mathrm{C}$ & $0.353(0.384)$ & $0.301(0.357)$ & $0.285(0.290)$ & $0.244(0.238)$ & $0.177(0.162)$ \\
$\mathrm{Z}_{2} \mathrm{~A}=\mathrm{B}^{+}-\mathrm{C}^{-}$ & $0.353(0.384)$ & $0.316(0.287)$ & $0.285(0.290)$ & $0.244(0.238)$ & $0.195(0.182)$ \\
$\mathrm{MI}^{-} \mathrm{A}-{ }^{+} \mathrm{B}^{+}-\mathrm{C}^{-}$ & $0.136(0.082)$ & $0.108(0.067)$ & $0.083(0.043)$ & $0.068(0.038)$ \\
$\mathrm{Z}_{3}{ }^{-} \mathrm{A}-\mathrm{B}-\mathrm{C}^{+}$ & & & & \\
$\mathrm{Z}_{4}{ }^{+} \mathrm{A}-\mathrm{B}-\mathrm{C}^{-}$ & & $0.049(0.033)$ & & \\
\hline
\end{tabular}

The BOVB method has several levels of sophistication. ${ }^{23,30}$ Here, we chose the "D-BOVB" level, whereby the orbitals that do not belong to the $\pi$-space are allowed to delocalize and are treated as doubly occupied. Thus, the VB treatment and the generation of $\mathrm{VB}$ structures are reserved to the $\pi$-system. This simplification is also applied to the VBSCF level. On the other hand, the $\pi$ AOs are each strictly localized on a single atom.

The VBSCF and BOVB calculations have been performed using the correlation-consistent triple- $\zeta$ cc-pVTZ basis set of Dunning. ${ }^{31}$ All VB calculations were performed by using the XMVB ab initio VB program of $\mathrm{Wu}$ and co-workers. ${ }^{32}$

\section{COMPUTATIONAL RESULTS}

Having four electrons to be distributed in three $\pi$ AOs, all 1,3dipoles $\mathrm{A}-\mathrm{B}-\mathrm{C}$ possess six VB structures, displayed in Scheme 2. Apart from the three $V B$ structures $D, Z_{1}$, and $Z_{2}$, discussed above, three other ones must be added to generate a complete and nonredundant basis of structures: ${ }^{23} \mathbf{Z}_{3}$ and $\mathbf{Z}_{4}$, in which one of the terminal atoms has a sextet structure, and $\mathrm{MI}$, a multi-ionic structure in which the central atom has a double positive charge.

The present VB calculations have been performed on ozone and its sulfur-substituted analogues using the optimized geometries taken from the accurate MRCI calculations of Xantheas et al. $^{20}$ All six structures were included in our preliminary VB calculation of VBSCF type, then structures having a negligible weight $(<0.01)$ were eliminated for the BOVB level, for the sake of avoiding computational instabilities. As a result of this selection criterion, the BOVB wave functions optimized for the different dimers contain three to six structures, depending on the cases, hence, the presence of some empty cells in Tables 1 and 2 , in which only values calculated at the BOVB level are reported.

The diabatic energies of the individual VB structures that are retained at the BOVB level are displayed in Table 1, taking the energy of the diradical structure as a reference. These diabatic energies are defined here as the diagonal elements of the Hamiltonian matrix of the optimized multistructure BOVB wave function. Restricting the discussion to the three most important $\mathrm{VB}$ structures $-\mathrm{D}, \mathrm{Z}_{1}$, and $\mathrm{Z}_{2}$-it can be seen that the six 1,3-dipoles display very contrasted relative diabatic energies. The diradical structure (D) is found to lie much lower than the zwitterionic ones $\left(\mathrm{Z}_{1}\right.$ and $\left.\mathrm{Z}_{2}\right)$ in $\mathrm{O}_{3}$, SOO, and SOS, with a gap between $\mathbf{D}$ and the lowest of $\mathrm{Z}_{1}$ and $\mathrm{Z}_{2}$ being larger than $40 \mathrm{kcal} / \mathrm{mol}$ and increasing in the order $\mathrm{O}_{3}<\mathrm{SOO}<\mathrm{SOS}$. At the other extreme, the diradical structure lies much higher in energy than the major zwitterionic ones in OSO, letting us predict a small and quite minor diradical character for this molecule. Lastly, the $\mathrm{D}, \mathrm{Z}_{1}$, and $\mathrm{Z}_{2} \mathrm{VB}$ structures are almost degenerate and, therefore, should contribute equally to the ground state in OSS and $\mathrm{S}_{3}$.

Table 1 also displays the total absolute energies of the ground states, resulting from the variational mixing of the individual VB structures for each molecule. Interestingly, the two pairs of isomeric 1,3-dipoles exhibit very large differences of stabilities, with OSS being more stable than SOS (by $62 \mathrm{kcal}$ $\mathrm{mol}^{-1}$ ) and OSO being more stable than SOO (by $109 \mathrm{kcal}$ $\mathrm{mol}^{-1}$ ). We will return to this important point below.

The weights of the various VB structures, as calculated at the BOVB level, are displayed in Table 2, according to the Chirgwin-Coulson formula (eq 11) and to the inverse weight formula (eq 12, weights given in parentheses).

It can be seen that the two definitions yield structural weights in very good agreement with each other, showing that, as expected and commented above, the ambiguity of having different definitions is not a real issue in the case of 1,3-dipoles where the $\pi$-system is the active one. Moreover, it can be seen, by comparing Tables 1 and 2, that, for each 1,3-dipole, the ordering of $\mathrm{VB}$ weights follows the ordering of diabatic energies: the lower the energy, the greater the weight, as any chemist would expect. There is only one minor exception, using the Chirgwin-Coulson definition, for OSS in which the $\mathrm{O}^{-}-$ $\mathrm{S}^{+}=\mathrm{S}$ structure is slightly lower than the $\mathrm{O}=\mathrm{S}^{+}-\mathrm{S}^{-}$one (by only $8.5 \mathrm{kcal} / \mathrm{mol}$ ), whereas the weights display a tiny difference in the reverse order $(30.1 \%$ vs $31.6 \%)$. On the other hand, even this insignificant exception disappears with the inverse weights. Therefore, both definitions of VB weights pass a first test of credibility. A much more demanding test, 


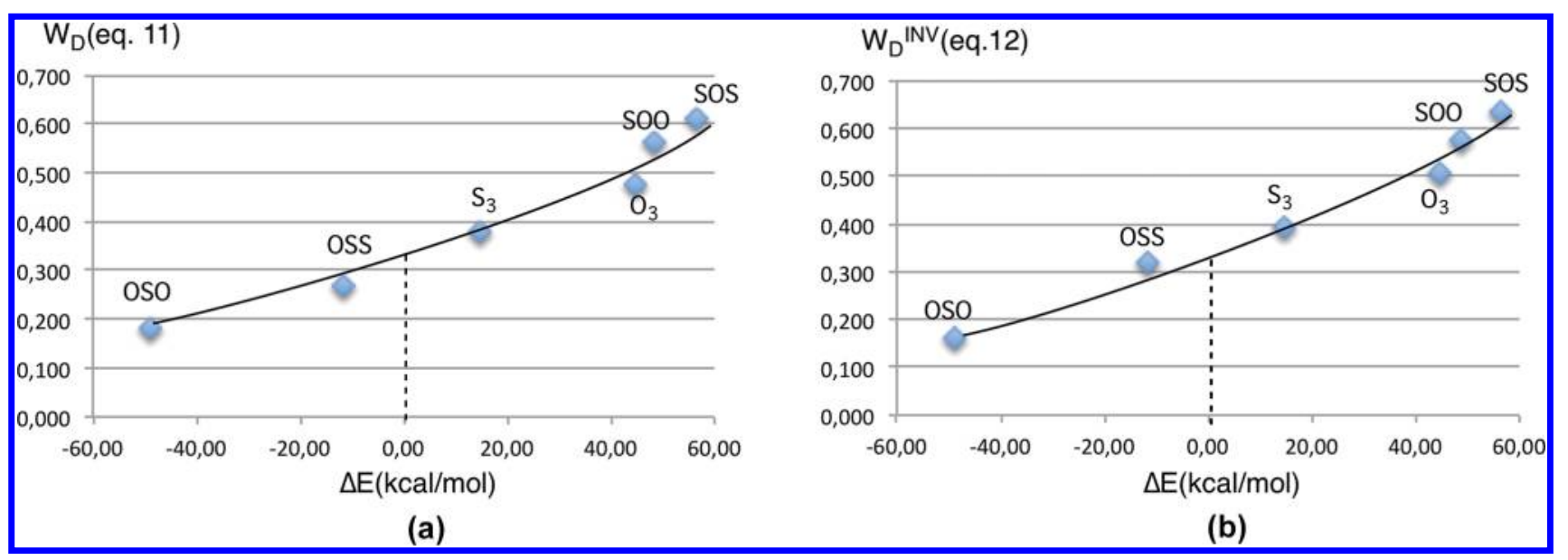

Figure 1. Renormalized diradical weights vs $E$ (lowest of $\mathbf{Z}_{1}$ and $\mathbf{Z}_{2}$ ) $-E(\mathbf{D}), \Delta E$ : (a) Chirwin-Coulson weights (eq 11) and (b) inverse weights (eq 12).

however, is the compliance of the VB weights of the diradical structures to eqs $13-15$. To check it, we have plotted the diradical weights against the energy gaps between $\mathbf{D}$ and the lowest of $\mathbf{Z}_{1}$ and $\mathbf{Z}_{2}$ for the six molecules. For making eqs 13-15 valid, we have only considered the three major structures and renormalized the weights accordingly. The results are displayed in Figures $1 \mathrm{a}$ and $1 \mathrm{~b}$, for the ChirgwinCoulson and inverse weights, respectively. Actually, the two curves are so similar that they can be discussed together. It can first be seen that the diradical weights smoothly increase along with the energy gaps, positive when $\mathbf{D}$ is the lowest-lying structure, negative when the lowest one is $\mathrm{Z}_{1}$ or $\mathrm{Z}_{2}$. For $\mathrm{O}_{3}$, SOO, and SOS, the diradical structure is the most stable one, lying some $45-57 \mathrm{kcal} \mathrm{mol}^{-1}$ below the zwitterionic ones. In accord, and in agreement with eq 14, their diradical weights are in the range of $44 \%-64 \%$, which is well over the $33 \%$ threshold. By contrast, the diradical structure is high-lying in OSO, $49 \mathrm{kcal} / \mathrm{mol}$ over the zwitterionic ones, and its weight is well below $33 \%$. OSS and $\mathrm{S}_{3}$ are borderline cases, with their diradical structure lying slightly lower and slightly higher than the zwitterionic ones, respectively. Accordingly, their weights are on both sides of the 33\% threshold and close to it. Finally, in both Figures $1 \mathrm{a}$ and $1 \mathrm{~b}$, the correlating curve crosses the vertical of point 0.0 of the abscissa, which corresponds to the hypothetical situation of $\mathrm{D}, \mathrm{Z}_{1}$, and $\mathrm{Z}_{2}$ being perfectly degenerate, at a diradical weight very close to the $33 \%$ threshold, as predicted by eq 16 .

\section{DISCUSSION}

Table 3 displays a comparison between the various values arising from different definitions of the diradical character of the 1,3-dipoles under study: the Chirgwin-Coulson and inverse VB-calculated weights (eqs 11 and 12), the $B_{\text {orb }}$ index based on $\pi$-AOs occupancies (eq 6), and the most recent definition based on the configuration coefficients, the $\beta$ index (eq 7). It can be seen, as already noted, that the ChirgwinCoulson and inverse weights are in very good agreement with each other, and also in agreement with an analysis that would be uniquely based on diabatic energies. Thus, SOO and SOS are best described as a first approximation as diradicals for two reasons that reinforce each other: (i) in each molecule, the diradical structure has a weight greater than $50 \%$, and (ii) this VB structure is much lower in energy than the other ones. On the other hand, the diradical structure has a small weight and
Table 3. Estimations of the Diradical Characters of 1,3Dipoles

\begin{tabular}{lcccc} 
& \multicolumn{3}{c}{ BOVB $^{a}$} \\
& \multicolumn{1}{c}{$\begin{array}{c}\text { Chirgwin-Coulson } \\
\text { weights (eq 11) }\end{array}$} & $\begin{array}{c}\text { inverse weights } \\
(\text { eq 12) }\end{array}$ & $B_{\text {orb }}{ }^{b}$ & $\beta^{c}$ \\
O-S-O & 0.159 & 0.150 & 0.086 & 0.035 \\
O-S-S & 0.227 & 0.257 & & 0.044 \\
S-S-S & 0.346 & 0.377 & 0.340 & 0.108 \\
O-O-O & 0.444 & 0.486 & 0.436 & 0.180 \\
S-O-O & 0.561 & 0.578 & & 0.260 \\
S-O-S & 0.610 & 0.635 & 0.626 & 0.353
\end{tabular}

${ }^{a}$ Data obtained in this work. ${ }^{b}$ Data taken from ref $19 .{ }^{c}$ Data taken from ref 20 .

lies high in energy in the OSO molecule, which is, therefore, well-described by as a first approximation by the usual textbook combination of $\mathrm{Z}_{1}$ and $\mathrm{Z}_{2}$ zwitterionic structures. Between these two extremes, the energy-based and VB-weight-based indicators agree to ascribe a strong diradical character to ozone and a significant but less important one to $S_{3}$ and OSS.

These very contrasted diradical characters of different 1,3dipoles have obvious consequences on their reactivities. As we have shown in a previous study, the barriers to cycloadditions of three families of 1,3-dipoles have an inverse correlation with the weights of their diradical structures. ${ }^{26}$ For the case at hand, this correlation is confirmed by the well-known fact that ozone is a very reactive species that reacts with virtually all $\mathrm{C}-\mathrm{C}$ multiple bonds, whereas OSO is unreactive. ${ }^{25}$ As already mentioned, this striking difference of reactivity also extends to the addition of $\mathrm{H}$ atoms to the terminal atoms, which is much easier for ozone than for OSO. ${ }^{12}$ Besides, the present results show that the relative stabilities of different isomers are also related to their diradical/closed-shell nature. Therefore, OSO and OSS are much more stable than their respective isomers, SOO and SOS, by as much as 109 and $62 \mathrm{kcal} \mathrm{mol}^{-1}$, respectively (see Table 1 ). This correlates very well with the strong diradical characters of SOO and SOS, which contrast with the much weaker ones in OSO and OSS: the higher the diradical weight, the less stable the isomer.

The $B_{\text {orb }}$ index (column 3 of Table 3 ) is found to be in fair agreement with the $\mathrm{VB}$ ones and to lead to the same qualitative conclusions, which is not surprising, since this index has a clear and well-justified physical basis, given that (i) the three VB 
structures $\mathbf{D}, \mathbf{Z}_{1}$, and $\mathbf{Z}_{2}$ have different $\pi$-AOs occupancies, and (ii) the $\pi$-AOs overlap weakly. Therefore, the $\mathrm{VB}$ weights can be deduced from a simple population analysis, at least in the simple case of 1,3-dipoles. These two features unfortunately, cannot be generalized to more-complicated systems.

Lastly, the $\beta$ index (eq 6; see column 4 in Table 3 ) gives systematically lower diradical characters than the other three. However, Figure 2 shows that this index smoothly varies along

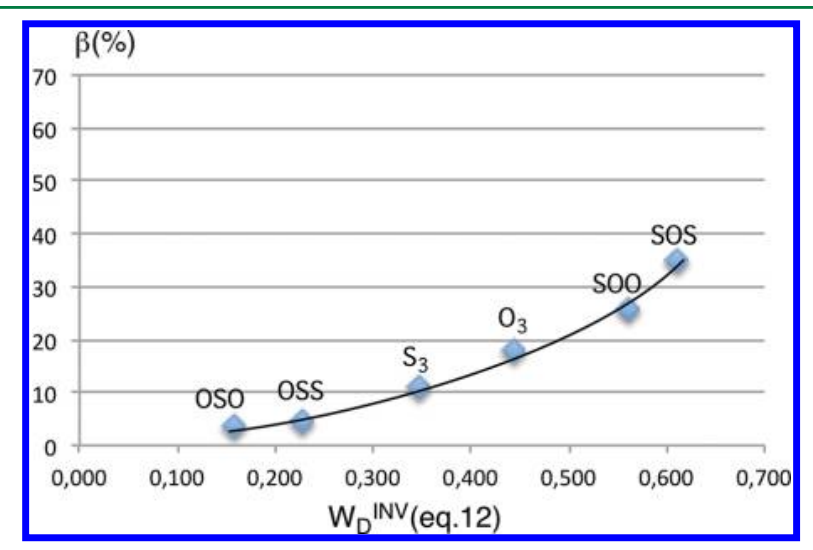

Figure 2. $\beta$ index (eq 7) vs inverse VB-calculated diradical weights (eq 12).

with the VB inverse weights (of course, a similar correlation is found with the Chirgwin-Coulson VB weights). Thus, the $\beta$ index can be taken as a useful qualitative indicator of diradical character, which displays correct tendencies within a series of molecules, with the advantage that this index is readily calculated with any standard ab initio MO-based method. However, this index cannot be taken as an absolute value that would quantitatively measure the weight of the diradical structure, to be compared with the other VB structures of the molecule. Such an overinterpretation would lead one to conclude, erroneously, that the diradical structure of ozone is minor, with a weight of only $18 \%$, whereas the zwitterionic structures $Z_{1}$ and $Z_{2}$ would share the remaining $82 \%$. This would be in contradiction with the diradical structure lying some $44 \mathrm{kcal} \mathrm{mol}^{-1}$ below the zwitterionic ones, according to accurate VB calculations.

It can be seen that our quantitative estimations of VB structure weights agree with the conclusions of Dunning et al., that both $\mathrm{O}_{3}$ and $\mathrm{SOO}$ have substantial diradical character, while OSO does not and is best described as a closed-shell structure. $^{10-12}$ More specifically, Dunning et al. described OSO, at the GVB level, with four AOs in the $\pi$-space, two $2 \mathrm{p}$ like AOs on the terminal oxygens, plus a pair of $\pi$-AOs on the central $S$ atom, which is composed of a $3 p$-like $\mathrm{AO}$ and a $3 \mathrm{~d}$ like one displaying a mutual overlap of 0.88 . The resulting GVB wave function was dominated by a structure displaying two $\mathrm{S}-$ $\mathrm{O} \pi$-bonds, called a recoupled pair bond dyad. ${ }^{10-12}$ This prompted us to test the possibility of a hypervalent structure in OSO that would display two $\mathrm{S}-\mathrm{O} \pi$-bonds: one using the $3 \mathrm{p}$ like sulfur $\mathrm{AO}$ and the other using a $3 \mathrm{~d}$-like one. Now, such a structure was found to lie some $184 \mathrm{kcal} / \mathrm{mol}$ above $\mathbf{Z}_{1}$ and $\mathbf{Z}_{2}$ at the VBSCF level, and, thus, be rather secondary, relative to the four classical structures that we have used in this study for OSO. However, this does not mean that our results disagree with the GVB description of OSO. Indeed, our wave function is a multistructure one, where each VB structure is built with strictly localized active orbitals, whereas GVB describes OSO by means of a unique VB structure, which, with its two $\mathrm{S}-\mathrm{O} \pi$ bonds linking semilocalized orbitals, implicitly includes and nicely summarizes the sum of $Z_{1}$ and $Z_{2}$ structures that we find to be the major ones in this molecule.

\section{CONCLUSION}

The diradical character is an important property of 1,3-dipoles and many other molecules, with a strong relationship to their reactivities. As such, this property has attracted much attention from theoreticians, who devised a variety of methods and formulas to quantify it in $\mathrm{MO}-\mathrm{CI}$ wave functions. However, the availability of the efficient nonorthogonal Valence Bond program, ${ }^{32-34}$ for computing high level ab initio classical VB wave functions, including both static and dynamic electron correlation, offers the opportunity to quantify the diradical character (or weight of a diradical structure) by dealing with VB wave functions that are directly expressed in terms of $\mathrm{VB}$ structures, thus avoiding the ambiguities associated with the interpretations of MO configurations in VB terms. Such methods allow the calculation of reliable values for both the diabatic energies and the weights of each individual VB structure.

The present $\mathrm{ab}$ initio VB calculations, applied to ozone and its sulfur-substituted isomers, provide two series of VB weights, according to the Chirgwin-Coulson and inverse definitions, which are each consistent with the ordering of diabatic energies: the lower the energy of a VB structure, the greater its weight. This logical weight-energy correspondence was already found in a previous VB study of other partially diradical molecules: $\mathrm{S}_{2} \mathrm{~N}_{2}, \mathrm{Se}_{2} \mathrm{~N}_{2}$, and $\mathrm{Te}_{2} \mathrm{~N}_{2}$. ${ }^{35}$

The VB calculated diradical weights amount to $15 \%-16 \%$, $23 \%-26 \%$, 35\%-38\%, 44\%-49\%, 56\%-58\%, and 61\%-64\% for OSO, OSS, $\mathrm{S}_{3}, \mathrm{O}_{3}$, SOO, and SOS, respectively, according to the two definitions. Thus, the controversial case of ozone is seen to have quite a significant diradical character, practically on par with the alternative description, in terms of interacting zwitterions. These weights are fully consistent with the different reactivities, stabilities, and other molecular properties of the six molecules.

Lastly, the particular case of 1,3-dipoles offers an additional way of checking the adequacy of the VB calculated diradical weights: since these molecules can be discussed in terms of only three major structures, simple chemical wisdom allows one to predict a threshold absolute value of $33 \%$ for the diradical weight when the three structures are degenerate, and a smaller (larger) value when the diradical structure is higher (lower) in energy than the zwitterionic ones. Both Chirgwin-Coulson and inverse definitions satisfy this logical requirement. Therefore, we may conclude that ab initio VB-calculated weights of diradical structures can be taken as absolute (or quantitative) measures of diradical characters, and not simply as mere indicators of tendencies.

\section{AUTHOR INFORMATION}

\section{Corresponding Authors}

*E-mail: benoit.braida@upmc.fr (B. Braïda).

*E-mail: philippe.hiberty@u-psud.fr (P. C. Hiberty).

\section{ORCID}

Sérgio E. Galembeck: 0000-0001-5018-4643

Philippe C. Hiberty: 0000-0002-7081-4654 


\section{Author Contributions}

The manuscript was written through contributions by all authors. All authors have given approval to the final version of the manuscript.

\section{Notes}

The authors declare no competing financial interest.

\section{ACKNOWLEDGMENTS}

Prof. Wei Wu and his colleagues in Xiamen University (China) are gratefully acknowledged for making their newest version of the ab initio Valence Bond XMVB 2.1 code available to us. S.E.G. thanks São Paulo Research Foundation (FAPESP) (Grant Nos. 2008/02677-0, 2014/50265-3, and 2015/01589-0) and Conselho Nacional do Desenvolvimento Científico e Tecnológico (CNPq) for financial support (Grant No. 304447/ 2010), and $\mathrm{CNPq}$ for a research fellowship (Grant Nos. 304393/2013-4 and 308254/2016-3).

\section{ABBREVIATIONS}

VBSCF = valence bond SCF; BOVB = breathing orbital valence bond; GVB = generalized valence bond; $\mathrm{SC}=$ spin-coupled

\section{REFERENCES}

(1) (a) Heusgen, R. On the Mechanism of 1,3-Dipolar Cycloadditions. A Replay. I. Org. Chem. 1968, 33, 2291-2297. (b) Huisgen, R. 1,3-Dipolar Cycloaddition Chemistry, Vol. 1; Padwa, A., Ed.; Wiley: New York, 1984; pp 1-176.

(2) Lan, Y.; Zou, L.; Cao, Y.; Houk, K. N. Computational Methods to Calculate Accurate Activation and Reaction Energies of 1,3-Dipolar Cycloadditions of 24 1,3-Dipoles. I.Phvs. Chem. A 2011, 115, 1390613920.

(3) Goddard, W. A.; Dunning, T. H.; Hunt, W. J.; Hay, P. J. Generalized Valence Bond Description of Bonding in Low-Lying States of Molecules. Acc. Chem. Res. 1973, 6, 368-376.

(4) Hay, P. J.; Dunning, T. H., Jr.; Goddard, W. A. Configuration Interaction Studies of $\mathrm{O}_{3}$ and $\mathrm{O}_{3}{ }^{+}$. Ground and Excited States. $\underline{\text { I. Chem. }}$. Phvs. 1975, 62, 3912-3924.

(5) Hay, P. J.; Dunning, T. H., Jr. Geometries and Energies of the Excited States of $\mathrm{O}_{3}$ from Abinitio Potential Energy Surfaces. $\underline{\text { I. Chem. }}$ Phvs. 1977, 67, 2290-2303.

(6) See, for instance: (a) Vollhardt, K. P. C.; Schore, N. Organic Chemistry: Structure and Function; W. H. Freeman and Company: New York, 6th Edition, 2011; p 538. (b) Carey, F. A.; Sundberg, R. J. Advanced Organic Chemistry, Part B: Reactions and Synthesis, 4th Edition; Kluwer Academic Publishers: Dordrecht, The Netherlands, 2002; p 788. (c) Klein, D. R. Organic Chemistry, 2nd Edition; John Wiley and Sons, Inc.: Hoboken, NJ, 2015; p 92 (ex 2.60). (d) Klein, D. R. Student Guide and Solutions Manual, 2nd Edition; John Wiley and Sons, Inc.: Hoboken, NJ, 2015; p 58.

(7) Laidig, W. D.; Schaefer, H. F. Large Multiconfiguration SelfConsistent-Field Wave Function for the Ozone Molecule. I. Chem. Phvs. 1981, 74, 3411-3414.

(8) Meredith, C.; Quelch, G. E.; Schaefer, H. F., III. Open-Chain and Cyclic Protonated Ozone: the Ground-state Potential-Energy Hypersurface. I. Am. Chem. Soc. 1991, 113, 1186-1193.

(9) Stanton, J. F.; Bartlett, R. J.; Magers, D. H.; Lipscomb, W. N. Highly Correlated Single Reference Studies of the $\mathrm{O}_{3}$ Potential Surface. Dissociation and Atomization Energies. Chem. Phvs. Lett. 1989, 163, 333-338.

(10) Lindquist, B. A.; Takeshita, T. Y.; Woon, D. E.; Dunning, T. H., Jr. Bonding in Sulfur-oxygen Compounds- $\mathrm{HSO} / \mathrm{SOH}$ and $\mathrm{SOO} /$ OSO: An Example of Recoupled Pair in Bonding. I. Chem. Theory Comput. 2013, 9, 4444-4452.

(11) Takeshita, T. Y.; Lindquist, B. A.; Dunning, T. H., Jr. Insigths into the Electronic Structure of Ozone and Sulfur Dioxide from
Generalized Valence Bond Theory: Bonding in $\mathrm{O}_{3}$ and $\mathrm{SO}_{2}$. I. Phvs. Chem. A 2015, 119, 7683-7694.

(12) Lindquist, B. A.; Takeshita, T. Y.; Dunning, T. H., Jr. Insights into the Electronic Structure of Ozone and Sulfur Dioxide from Generalized Valence Bond Theory: Addition of Hydrogen Atoms. I. Phvs. Chem. A 2016, 120, 2720-2726.

(13) Kalemos, A.; Mavridis, A. Electronic Structure and Bonding in Ozone. I. Chem. Phvs. 2008, 129, 054312.

(14) Arnold, D. W.; Xu, C.; Kim, E. H.; Neumark, D. M. Study of Low-Lying Electronic States of Ozone by Anion Photoelectron Spectroscopy of $\mathrm{O}^{-}$. I. Chem. Phvs. 1994, 101, 912-922.

(15) Woywod, C.; Stengle, M.; Domcke, W.; Flöthmann, H.; Schinke, R. Photodissociation of Ozone in the Chappuis Band. I. Electronic Structure Calculations J. I. Chem. Phys. 1997, 107, 72827295.

(16) (a) Cooper, D. L.; Gerratt, J.; Raimondi, M. Raimondi Applications of Spin-Coupled Valence Bond Theory. Chem. Rev. 1991, 91, 929-964. (b) Gerratt, J.; Cooper, D. L.; Karadakov, P. B.; Raimondi, M. Spin-Coupled Theory; The Encyclopedia of Computational Chemistry; John Wiley \& Sons, Ltd.: Chichester, U.K., 1998.

(17) (a) Penotti, F. E.; Cooper, D. L. Combining Rival $\pi$-Space descriptions of $\mathrm{O}_{3}$ and $\mathrm{SO}_{2}$. Int. I. Quantum Chem. 2016, 116, 718730. (b) Thorsteinsson, T.; Cooper, D. L.; Gerratt, J.; Karadakov, P. B.; Raimondi, M. Modern Valence Bond Representation of CASSCF Methods. Theor. Chim. Acta 1996, 93, 343-366.

(18) Tuononen, H. M.; Suontamo, R.; Valkonen, J.; Laitinen, R. S. Electronic Structure and Spectroscopic Properties of $6 \pi$-Electron Ring Molecules and Ions $\mathrm{E}_{2} \mathrm{~N}_{2}$ and $\mathrm{E}_{4}{ }^{2+}(\mathrm{E}=\mathrm{S}, \mathrm{Se}, \mathrm{Te})$. L.Phvs. Chem. A 2004, 108, 5670-5677.

(19) Glezakou, V. A.; Elbert, S. T.; Xantheas, S. S.; Ruedenberg, K. Analysis of Bonding Patterns in the Valence Isoelectronic Series $\mathrm{O}_{3}, \mathrm{~S}_{3}$, $\mathrm{SO}_{2}$, and $\mathrm{OS}_{2}$ in Terms of Oriented Quasi-atomic Molecular Orbitals. I. Phys. Chem. A 2010, 114, 8923-8931.

(20) Miliordos, E.; Xantheas, S. S. Analogues $\mathrm{SO}_{2}, \mathrm{OS}_{2}$ and $\mathrm{S}_{3}$ : Correlation between their Biradical Character and Molecular Properties. I. Am. Chem. Soc. 2014, 136, 2808-2817.

(21) Salem, L.; Rowland, C. The Electronic Properties of Diradicals. Angew. Chem., Int. Ed. Engl. 1972, 11, 92-111.

(22) Miliordos, E.; Ruedenberg, K.; Xantheas, S. S. Unusual Inorganic Biradicals: A Theoretical Analysis. Angew. Chem. Int. Ed. 2013, 52, 5736-5739.

(23) Shaik, S.; Hiberty, P. C. A Chemist's Guide to Valence Bond Theory; Wiley-Interscience: New York, 2008; pp 91-93.

(24) Hiberty, P. C.; Ohanessian, G. Comparison of Minimal and Extended Basis Sets in Terms of Resonant Formulas. Application to 1,3 Dipoles. I. Am. Chem. Soc. 1982, 104, 66-70.

(25) Lan, Y.; Wheeler, S. E.; Houk, K. N. Extraordinary Difference in Reactivity of Ozone (OOO) and Sulfur Dioxide (OSO). I. Chem. Theorv Comput. 2011, 7, 2104-2111.

(26) Braida, B.; Walter, C.; Engels, B.; Hiberty, P. C. A Clear Correlation Between the Diradical Character of 1,3-Dipoles and Their Reactivity Toward Ethylene and Acetylene. I. Am. Chem. Soc. 2010, 132, 7631-7637.

(27) (a) van Lenthe, J. H.; Balint-Kurti, G. G. The Valence-Bond SCF (VB SCF) Method: Synopsis of Theory and Test Calculation of the OH Potential Energy Curve. Chem. Phvs. Lett. 1980, 76, 138-142. (b) van Lenthe, J. H.; Balint-Kurti, G. G. The Valence Bond SelfConsistent Field Method (VB-SCF): Theory and Test Calculations. I. Chem. Phvs. 1983, 78, 5699-5713.

(28) Chirgwin, B. H.; Coulson, C. A. The Electronic Structure of Conjugated Systems. VI. Proc. R. Soc. London, Ser. A 1950, 201, 196209.

(29) Gallup, G. A.; Norbeck, J. M. Population Analyses of ValenceBond Wavefunctions and $\mathrm{BeH}_{2}$. Chem. Phvs. Lett. 1973, 21, 495-500.

(30) (a) Hiberty, P. C.; Flament, J. P.; Noizet, E. Compact and Accurate Valence Bond Functions with Different Orbitals for Different Configurations - Applications to the 2-Configuration Description of $\mathrm{F}_{2}$. Chem. Phvs. Lett. 1992, 189, 259-265. (b) Hiberty, P. C.; Humbel, S.; Byrman, C. P.; van Lenthe, J. H. Compact Valence-Bond Functions 
with Breathing Orbitals-Application to the Bond-Dissociation Energies of $F_{2}$ and FH. I. Chem. Phvs. 1994, 101, 5969-5676. (c) Hiberty, P. C.; Shaik, S. Breathing-Orbital Valence Bond Method-A Modern Valence Bond Method that Includes Dynamic Correlation. Theor. Chem. Acc. 2002, 108, 255-272.

(31) Dunning, T. H., Jr. Gaussian-Basis Sets for Use in Correlated Molecular Claculations. 1. The Atoms Boron through Neon and Hydrogen. I. Chem. Phvs. 1989, 90, 1007-1023.

(32) (a) Song, L.; Chen, Z.; Ying, F.; Song, J.; Chen, X.; Su, P.; Mo, Y.; Zhang, Q.; Wu, W., XMVB 2.1: An ab initio Non-Orthogonal Valence Bond Program, Xiamen University, Xiamen, China, 2015. (b) Song, L.; Mo, Y.; Zhang, Q.; Wu, W. J. I. Comput. Chem. 2005, 26, 514. (c) Chen, Z.; Chen, X.; Wu, W. I. Chem. Phys. 2013, 138, 164120. (d) http://www.xmvb.org/.

(33) Verbeek, J.; Langenberg, J. H.; Byrman, C. P.; Dijkstra, F.; Engelberts, J. J.; Zielinski, M.; van Lenthe, J. H. TURTLE, an ab initio VB/VBSCF program. Utrecht, The Netherlands, 1988-2015, implemented in GAMESS-UK (see ref 34).

(34) Guest, M. F.; Bush, I. J.; van Dam, H. J. J.; Sherwood, P.; Thomas, J. M. H.; van Lenthe, J. H.; Havenith, R. W. A.; Kendrick, J. Mol. Phvs. 2005, 103, 719-747.

(35) Braida, B.; Lo, A.; Hiberty, P. C. Can Aromaticity Coexist with Diradical Character? An Ab Initio Valence Bond Study of $\mathrm{S}_{2} \mathrm{~N}_{2}$ and $\mathrm{E}_{4}{ }^{2+}(\mathrm{E}=\mathrm{S}, \mathrm{Se}, \mathrm{Te})$. ChemPhysChem 2012, 13, 811-819. 